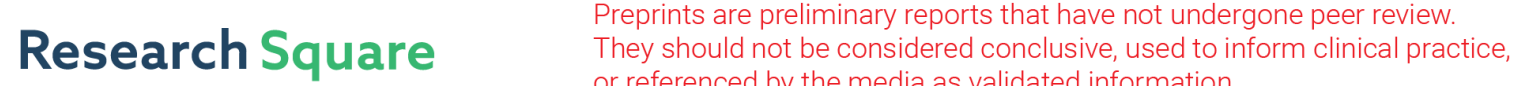 or referenced by the media as validated information. \\ Identification of ferroptosis-related genes for overall survival prediction in hepatocellular carcinoma
}

Lianxiang Luo ( $\square$ luolianxiang321@gdmu.edu.cn )

Guangdong Medical University

Xinyue Yao

Guangdong Medical University

Fangfang Huang

Guangdong Medical University

Hui Luo

Guangdong Medical University

\section{Research Article}

Keywords: hepatocellular carcinoma, ferroptosis, immune cells, drug target genes

Posted Date: May 17th, 2021

DOI: https://doi.org/10.21203/rs.3.rs-523396/v1

License: @ (i) This work is licensed under a Creative Commons Attribution 4.0 International License.

Read Full License 


\title{
Identification of ferroptosis-related genes for overall survival prediction in hepatocellular carcinoma
}

\author{
Lianxiang Luo ${ }^{1,2,3 *}$, Xinyue $\mathrm{Yao}^{4}$, Fangfang Huang ${ }^{5}$, Hui Luo $\mathrm{Lu}^{1,2,3^{*}}$ \\ ${ }^{1}$ Southern Marine Science and Engineering Guangdong Laboratory (Zhanjiang), Zhanjiang, \\ Guangdong, 524023, China \\ ${ }^{2}$ The Marine Biomedical Research Institute, Guangdong Medical University, Zhanjiang, Guangdong, \\ 524023, China \\ ${ }^{3}$ The Marine Biomedical Research Institute of Guangdong Zhanjiang, Zhanjiang, Guangdong, 524023, \\ China \\ ${ }^{4}$ The First Clinical College, Guangdong Medical University, Zhanjiang, Guangdong, 524023, China \\ ${ }^{5}$ Graduate School, Guangdong Medical University, Zhanjiang, Guangdong, 524023, China \\ * Corresponding authors, Email: 1uolianxiang321@gdmu.edu.cn; luohui@gdmu.edu.cn;
}

\begin{abstract}
Ferroptosis is a novel type of cell death depending on iron, which has been confirmed strongly related with the development of tumor. Hepatocellular carcinoma (HCC) is a malignancy with high incidence. Despite some reports demonstrated the relation between ferroptosis-related genes and HCC, more details have not been excavated. In present study, we analyzed ferroptosis-related genes with their clinical information from TCGA-LIHC project to find out prognostic genes in HCC. And four genes (GPX2, MT3, PRDX1 and SRXN1) were established as a prognostic model after differentially expressed analysis, Cox regression analyses and LASSO approach. High-risk group separating by cut-off value with poor prognosis was proved, and risk score regarded as an independent prognostic factor. Subsequently, enrichment analyses were processed to the differentially expressed genes, we found that genes generally enriched in ferroptosis-related functions and pathways, also in some immune cells and functions with different immune status. Eventually, we constructed ferroptosis potential index (FPI) to reveal the functional roles of ferroptosis in tumor tissues. The immunohistochemistry performed prognostic genes expression in normal and tumor tissues. In conclusion, these results demonstrated the four-gene signature can be a biomarker for predicting HCC prognosis and its members can be drug target genes for HCC treatment.
\end{abstract}

\section{Introduction}

Hepatocellular carcinoma (HCC) is one of the most common heterogeneous malignancies, which is the sixth most widespread neoplasm and the fourth leading cause of cancer death ${ }^{1}$. The incidence and mortality of liver cancer is gradually increasing, and HCC is accounting for $\sim 90 \%$ of primary liver cancers $^{1}$. Concerning the complex pathogenesis of HCC, some reports about the etiology and development of HCC have speculated that it is associated with cirrhosis, viral hepatitis, specific chemical carcinogens and abnormal regulation of hormones ${ }^{2}$. However, early diagnosis and treatment of HCC remain an issue, especially in the low-level medical developing countries ${ }^{3}$. Although the diagnostic and therapeutic techniques for HCC are developing rapidly ${ }^{4}$, the prognosis of HCC is very poor, approximately $70 \%$ of $\mathrm{HCC}$ relapse within 5 years after receiving resection or ablation ${ }^{5}$. Currently, due to the not obvious early clinical symptoms, most of the patients were firstly diagnosed in the middle or late stages so that they miss the optional treatment period. Consequently, it needs more 
effort to identify early diagnostic biomarkers of HCC, and their utilization are beneficial for timely and effective treatment for patients.

Ferroptosis is an iron-independent form of cell death, the features and mechanisms are different from apoptosis, necrosis, and autophagy, which is characterized by the accumulation of lipid reactive oxygen species (ROS) ${ }^{6}$. The cystine/glutamate antiporter (system Xc-) and Glutathione peroxidase-4 (GPX4) in the typical glutathione pathway are two vital regulatory points of ferroptosis mechanisms ${ }^{7}$. The system $\mathrm{Xc}^{-}$can regulate the exchange of intracellular glutamate and extracellular cystine ${ }^{8}$. And GPX4, as a unique member of selenium-dependent glutathione peroxidase distributed within mammals, plays a pivotal role in suppressing the generation of lipid ROS during ferroptotic cell death ${ }^{9}$. Interestingly, cysteine impedes the biosynthesis of glutathione (GSH), which is a substrate of GPX4. Therefore, the interaction of the system $\mathrm{Xc}^{-}$and GPX4 induces the accumulation of ROS and ferroptosis ${ }^{6}$. Sorafenib, as the standard first-line drug against advanced $\mathrm{HCC}$, could inhibit system $\mathrm{Xc}^{-}$and induce the ferroptosis to exert its cytotoxic effects ${ }^{10-12}$. Some previous studies have also reported the importance of ferroptosis for the treatment and prognosis of liver cancer $^{13-16}$, but the hub ferroptosis-related regulators and potential regulatory mechanism of ferroptosis during the occurrence and progression of HCC have not been investigated in detail.

In this study, we systematically analyzed some ferroptosis-related genes in HCC based on the TCGA (The Cancer Genome Atlas, https://portal.gdc.cancer.gov/repository) datasets and try to clarify how they affect the pathogenesis and prognosis of HCC. We hope that the biomarkers will be helpful for the diagnosis, treatment, and prognosis of HCC.

\section{Results}

\section{Identification of differentially expressed FRGs}

A flow chart was performed to completely describe our study (Fig. 1). The training cohort was obtained from TCGA-LIHC project, which contained 374 tumor samples and 50 normal samples. Meanwhile, we acquired 214 ferroptosis-related genes (FRGs) from FerrDb database (http://www.zhounan.org/ferrdb) that including 98 drivers, 94 suppressors and 101 makers. Intersecting two datasets to get a list of 200 ferroptosis-related genes with its expression profile in TCGA-LIHC cohort. The "limma" R package was used to analyze the expression pattern of FRGs. Totally, the analysis identified 126 differentially expressed FRGs, including 110 high expressed genes and 16 low expressed genes (Fig. 2A). Then, we tried to describe the overall condition about the simple nucleotide variation for 126 differentially expressed genes (DEGs). And we found that the variant classification and variant type between up-regulated genes and down-regulated genes have the same distributions (Fig. 2B, C). To investigate the correlation of all the DEGs, make an analysis of these DEGs by STRING website (https://string-db.org/). The result, a PPI network, was visualized by Cytoscape software (version 3.8.2), and the orange means high expression but the blue means low expression. (Fig. 2D).

\section{Establishment and assessment of prognostic model}

To understand what role FRGs play in the HCC more deeply, we applied univariate Cox regression analysis and received 19 prognostic genes (Table 1). The LASSO (Least Absolute Shrinkage and Selection Operator) regression analysis was used to get the genes which are obviously related to overall survival of HCC (Fig. 3A), and a cross-validation for the model of selected genes is necessary (Fig. 3B). For a stable prognosis-related gene signature, the 19 genes related to overall survival (OS) was 
processed by stepwise multivariate Cox regression analysis after LASSO approach. Following that, we finally built a four-gene signature for overall survival in HCC, and the four genes are GPX2, MT3, PRDX1 and SRXN1 (Fig. 3C). According to the multivariate Cox regression coefficients, the risk score of each sample in training cohort was calculated. To assure the accurate separating standard of the risk score group, we painted an ROC curve to search cut-off value based on the risk score, and the cut-off value we got is 1.275 (Fig. 4A). The 167 HCC patients in training dataset were divided into two groups, high- $(n=25)$ and low-risk group $(n=142)$, based on the cut-off value. Comparing two groups with its survival status and the four genes expression, the high-risk group is obviously associated with a comparative high death rate and with higher gene expression (Fig. 3D-F). Through the same process, the HCC patients in validation cohort were divided into high- $(n=92)$ and low-risk group $(n=43)$ by cut-off value (cut-off value $=0.802$ ) (Fig. 4D). And the results that the correlation in risk score and survival status even genes expression are similar to the training set (Fig. 3G-I).

Next, we applied Kaplan-Meier analysis to perform the difference of survival status between high-risk group and low-risk group in training cohort (Fig. 4B). The Kaplan-Meier curve presents the significant difference between high- and low-risk group $(\mathrm{P}<0.0001)$. For a better convincing, we painted the ROC curve to validate the result of Kaplan-Meier curve, and a higher AUC (Area Under Curve) means a better performance. The AUCs of OS corresponding to 1, 2 and 3 years in training set were 0.682 , 0.694 and 0.539 , respectively (Fig. 4C). The two plots were also validated in testing cohort, and we got the same result that Kaplan-Meier curve performed a differentially expressed OS between two groups $(\mathrm{P}=0.025)$ (Fig. 4E), AUCs were $0.534,0.681$ and 0.635 respectively contacting to the OS of 1,2 and 3 years in validation set (Fig. 4F). The credible AUCs can prove high-risk group with poor prognosis presented by Kaplan-Meier curve in HCC patients.

For the prognostic model, univariate Cox regression analysis and multivariate Cox regression analysis were applied to assess the clinical characteristics whether are the independent predictively prognostic factors or not. As shown in Figure 5, with four clinical parameters (Age, Gender, Stage and risk score), it is suggested that risk score is the most significant clinical feature related to survival in training and testing cohorts $(\mathrm{P}<0.05)$, but the $\mathrm{p}$-value of risk score processed by multivariate Cox regression analysis in ICGC (International Cancer Genome Consortium, https://dcc.icgc.org/projects/LIRI-JP) dataset $(\mathrm{P}=0.053)$ is a few more than 0.05 . Hence, we considered that risk score can be an independent prognostic predictor with high probability.

\section{Association between FRGs and immune}

To clearly evaluate the proportion of 19 tumor-infiltrating immune cells in each sample, CIBERSORT analysis was applied to HCC samples which p-value $<0.05$ in training dataset (Fig. 6A). Besides, we explored the correlation between risk score and immune status including immune cells and immune functions. Single-sample Gene Set Enrichment Analysis (ssGSEA) was applied to assess the abundance level of immune cells and immune functions in two cohorts (Fig. 6B-E), most of enrichment scores expressed highly in high-risk group. The Figure $6 \mathrm{~B}$ and Figure $6 \mathrm{C}$ presented the different enrichment scores of 5 immune cells and 4 immune functions between high- and low-risk groups in training dataset. And the enrichment score of neutrophils in 5 immune cells was significantly different in two risk groups $(\mathrm{P}<0.05)$, the enrichment scores of check-point and Para inflammation in 4 immune functions indicated significant difference $(\mathrm{P}<0.01)$. As shown in Fig. 6D and Fig. 6E, they presented different enrichment scores of 4 immune cells and 3 immune functions between high- and low-risk groups in validation dataset. However, the results were different from those in training cohort. The enrichment 
score of Treg cells in 4 immune cells was significantly different in two risk groups $(\mathrm{P}<0.05)$, but there were not obviously different enrichment scores about immune functions between high- and low-risk groups in testing cohort.

\section{Gene Ontology and Kyoto Encyclopedia of Genes and Genomes pathway enrichment analyses}

Subsequently, we applied functional enrichment analyses to the DEGs in high- and low-risk groups in training cohort. As observed, the result of functional annotation encompassing biological processes (BP), cellular components (CC) and molecular functions (MF) by Gene Ontology (GO) analysis performed some ferroptosis-related function, such as cellular response to oxidative stress in biological processes, antioxidant activity, oxidoreductase activity, acting on $\mathrm{NAD}(\mathrm{P}) \mathrm{H}$ in molecular functions and so on (Fig. 7A). In addition, the ferroptosis-related DEGs were dealt with Kyoto Encyclopedia of Genes and Genomes (KEGG) pathway enrichment analysis (Fig. 7B). As our expected, the pathway was significantly enriched on ferroptosis pathway. To guarantee the result more accurate, ICGC cohort, a validation dataset was accepted with GO and KEGG pathway enrichment analyses (Fig. 7C, D), too. Delightfully, the functions and pathways of enrichment in validation dataset was as same as the training dataset, especially the enriched pathways found by KEGG analysis that the first enrichment pathway was ferroptosis.

\section{The correlation between FPI and tissues and immunohistochemistry for validation}

To understand the influence on prognosis and therapy ferroptosis brought in HCC, ferroptosis potential index (FPI) quantizing the expression level of ferroptosis was established to reveal the ferroptotic functions and different expression between normal and tumor tissues in $\mathrm{HCC}$ patients from TCGA-LIHC cohort. As shown in Fig. 8A, the expression level of FPI has obvious difference ( $\mathrm{P}=$ 1.0e-04) in normal and tumor groups.

Finally, we found out the figures of immunohistochemistry (IHC) about the expression of encoding proteins by four-gene signature from The Human Protein Atlas (HPA) database. Obviously, for the expression level, PRDX1 strongly expressed in normal tissues, but GPX2 moderately expressed in tumor tissues (Fig. 8B-E). Regrettably, there were not IHC plots of MT3 and SRXN1 that can be completely compared with on the website.

\section{Discussion}

By the increasingly developed anticancer therapy of selective induction of cancer cell death, ferroptosis was received with great concern because of its special modality of cell death. Several reports have indicated that ferroptosis play a crucial role in the process of tumorigenesis ${ }^{17}$, 18 . Hepatocellular carcinoma has strong relation with metabolic disorders ${ }^{19}$. ROS has been proved to be chemically reactive metabolites containing oxygen that decrease in liver cancer ${ }^{20}$. Lipid ROS accumulating too much by exogenous drugs and iron-metabolism dysregulation both are the keys in ferroptosis origin and development ${ }^{6,21}$. There are several previous studies had explored the relation between ferroptosis-related genes and HCC prognosis, and received a few genes related to HCC prognosis ${ }^{22-24}$. However, the mechanism that how ferroptosis influence the tumor cell death in HCC patients and which kind of role the predictors for prognosis play is not demonstrated completely until now. Hence, our study constructed a prognostic model with four ferroptosis-related genes through bioinformatic methods, which was proved to be beneficial for early diagnosing to HCC patients.

In the present study, the prognostic model contained four genes_- GPX2, MT3, PRDX1 and SRXN1. 
Glutathione peroxidase 2 (GPX2), a member of the antioxidant enzyme GPX family, overexpression can induce poor prognosis of hepatocellular carcinoma patients ${ }^{25}$. Metallothionein III (MT3) overexpression contributes to carcinogenesis and poor prognosis of several other type of cancer patients but not clearly for HCC patients ${ }^{26}$. And with the downregulation of MT3 often accompanying to the methylation, there was a speculation that MT3 may suppress the tumor via promoting hypermethylation ${ }^{27}$. Up-regulated peroxiredoxin 1 (PRDX1) often acts as an oncogene in many type of cancer but remains controversial ${ }^{28}$. However, it was confirmed that overexpressed PRDX1 was closely related with poor prognosis of HCC patients ${ }^{29}$. Sulfiredoxin-1 (SRXN1) was revealed a pro-tumorigenic in $\mathrm{HCC}$ by regulating ROS signaling ${ }^{30}$. In addition, SRXN1 is involved in oxidoreductase activity ${ }^{31}$, its overexpression may play a crucial role in the tumorigenesis and progression of $\mathrm{HCC}^{32}$. Nevertheless, there are not so many reports about the relation between these genes and ferroptosis. We can only know that the expression of GPX2, MT3 and SRXN1 were upregulated during ferroptosis induced by erastin or RSL3, and they may promote ferroptosis 7 . Besides, PRDX1 is necessary to ferroptosis-related lipid peroxidation, and it also may promote ferroptosis because ferroptosis-related suppressor can block enhanced lipid peroxidation and recover cell viability without PRDX1 ${ }^{33}$. After constructing the four-gene signature, none of the clinical parameters were proved to be an independent prognostic factor except risk score.

Then we conducted a series of enrichment analyses on functions and pathways. Based on the close association between ferroptosis and oxidative stress ${ }^{34}$, we observed the functions of cellular response to oxidative stress, oxidoreductase activity, acting on $\mathrm{NAD}(\mathrm{P}) \mathrm{H}$ and so on were related with ferroptosis in two datasets. Interestingly, the depletion of NADPH will promote lipid ROS accumulation by reducing the GSH which metabolism can directly influence ferroptosis sensitivity ${ }^{34}$, ${ }^{35}$. Moreover, the KEGG pathway analysis indicated that glutathione metabolism and ferroptosis pathways were enriched, which suggested that all DEGs were strongly linked with ferroptosis. The immune score estimating by ssGSEA evaluated the abundances of the immune signature in risk groups. Besides, we also used ssGSEA to construct FPI to characterize ferroptosis based on genes expression pattern and the FPI is higher in tumor tissues. And FPI was put forward to model ferroptosis level which can predict poor prognosis with high score in many cancer types ${ }^{36}$.

In summary, we established a prognostic model with four ferroptosis-related genes through statistical analyses and processes another time in testing cohort to evaluate its accuracy. Meanwhile, we check the functions and pathways of genes to validate the connection between genes and ferroptosis. It can also demonstrate that the ferroptosis-related gene signature can be a predictor in HCC, linking ferroptosis and HCC more closely. However, the limitation in this study need improvement. The datasets we acquired both are from public databases, the quantity of samples and the completeness of clinical information need to collect more to strengthen its reliability. And we also need forward-looking study to further improve the availability of prognostic model.

\section{Methods}

\section{Data acquisition}

Expression RNA-seq (FPKM value), simple nucleotide variation data and clinical information of patients were obtained from the Cancer Genome Atlas (TCGA) (https://portal.gdc.cancer.gov/repository) dataset as a training cohort. The validation cohort was downloaded from ICGC portal (https://dcc.icgc.org/projects/LIRI-JP). A list of 214 ferroptosis-related genes (FRGs) was acquired from FerrDb database (http://www.zhounan.org/ferrdb), a web-based 
database of ferroptosis regulators and markers and ferroptosis-disease associations ${ }^{37}$.

\section{Differential expression analysis}

To identify differentially expressed genes (DEGs) between tumor tissues and normal tissues, we used "limma" package in R (version 4.0.2) to calculate the $\operatorname{logFC}$ (log fold change) and p-values of 214 ferroptosis-related genes. The DEGs were filtered using adjusted p-value (adjusted by false discovery rate $)<0.05$ and absolute $\log \mathrm{FC}>0.5$. Following this, the DEGs were separated into two groups (high-expressed and low-expressed groups) for simple nucleotide variation analysis. The "maftools" $\mathrm{R}$ package was used to analyze the summary of DEGs mutation information in TCGA dataset. Besides, we utilized the Search Tool for Recurring Instances of Neighbouring Genes (STRING) database (https://string-db.org/) to analyze a PPI network of DEGs, and then the PPI was visualized by Cytoscape software (version 3.8.2).

\section{Identification of prognostic FRGs and signature building}

A univariate Cox regression analysis was used to screen differentially expressed FRGs associated with overall survival (OS) in HCC patients, and we considered p-value $<0.05$ as statistical significance. To narrow the range of potential prognostic FRGs, we did a LASSO regression analysis by applying the "glmnet" package in R. Then, for performing the prognostic signature, we conducted the stepwise multivariate Cox regression analysis and constructed a ferroptosis-related four-gene signature. The prognostic risk score was calculated according to the expression levels of the genes and a linear combination of the regression coefficient $(\lambda)$ in a multivariate Cox regression model. The formula was established as follows: score $=$ sum (each gene's expression level $\times \lambda$ ). The patients were divided into high-risk group and low-risk group based on the cut-off value of the risk scores.

\section{Statistical analysis}

Using "survival", "survminer" and "timeROC" packages in R software to plot Kaplan-Meier survival curves and ROC curves, which can evaluate the potentially predictive performances of the prognostic signature. Finally, we conducted univariate and multivariate Cox regression analysis to confirm which traditional clinical characteristic was independent. Hazard ratios (HRs) and $95 \%$ confidence intervals (CIs) for each variable were calculated. P-value $<0.05$ was considered statistical significance.

\section{Immune cells and functions}

Cell-type Identification By Estimating Relative Subsets Of RNA Transcripts (CIBERSORT) analysis was used to evaluate the proportion of 19 human immune cell subpopulations by calculating the absolute abundance of immune cells and stromal cells. P-value $<0.05$ indicates statistical significance. Making single-sample GSEA (ssGSEA) to estimate the immune scores of immune cells and abundance of immune functions in two risk groups by using "GSVA" R package. Mann-Whitney test with p-value validated its differential expression between high-risk group and low-risk group.

\section{Functional enrichment analysis}

To explore the functional annotation which was associated with the risk score, we conducted Gene Ontology (GO) and Kyoto Encyclopedia of Genes and Genomes (KEGG) analyses based on the DEGs (FDR $<0.05$ ) by applying the "clusterProfiler" package in R software. P-values were adjusted with the FDR method. Next, we constructed the ferroptosis potential index (FPI) by using ssGSEA to reveal the functional roles of ferroptosis and differential FPI expression in tumor and normal tissues. Finally, we 
obtained the immunohistochemistry images of prognostic signature from HPA website (The Human Protein Atlas, https://www.proteinatlas.org/).

\section{Funding}

This project was supported by the PhD Start-up Fund of Guangdong Medical University (B2019016); Administration of Traditional Chinese Medicine of Guangdong Province (20201180); Administration of Traditional Chinese Medicine of Guangdong Province (20211223); Science and Technology Special Project of Zhanjiang (2019A01009); Basic and Applied Basic Research Program of Guangdong Province (2019A1515110201); Key Program of Marine Economy Development(Six Marine Industries) Special Foundation of Department of Natural Resources of Guangdong Province(GDNRC[2020]038); Fund of Southern Marine Science and Engineering GuangdongLaboratory (Zhanjiang) (ZJW-2019-007). Discipline Construction Project of Guangdong Medical University (4SG21004G)

\section{Conflicts of Interest}

The authors declare that they have no conflicts of interest in this study.

\section{Authorship contributions}

LX Luo conceived and designed the study; XY Yao performed the data analysis; XY Yao, FF Huang and LX Luo wrote the manuscript; LX Luo and H Luo reviewed the paper and provided comments, and all the authors reviewed the manuscript.

\section{References}

1. Bray, F. et al. Global cancer statistics 2018: GLOBOCAN estimates of incidence and mortality worldwide for 36 cancers in 185 countries. CA Cancer J Clin 68, 394-424 (2018).

2. Maluccio, M.\& Covey, A. Recent progress in understanding, diagnosing, and treating hepatocellular carcinoma. CA Cancer J Clin 62, 394-9 (2012).

3. Forner, A., Reig, M.\& Bruix, J. Hepatocellular carcinoma. Lancet 391, 1301-1314 (2018).

4. Dimitroulis, D. et al. From diagnosis to treatment of hepatocellular carcinoma: An epidemic problem for both developed and developing world. World J Gastroenterol 23, 5282-5294 (2017).

5. Cancer Genome Atlas Research Network. Electronic address, w.b.e.\& Cancer Genome Atlas Research, N. Comprehensive and Integrative Genomic Characterization of Hepatocellular Carcinoma. Cell 169, 1327-1341 e23 (2017).

6. Dixon, S.J. et al. Ferroptosis: an iron-dependent form of nonapoptotic cell death. Cell 149, 1060-72 (2012).

7. Yang, W.S. et al. Regulation of ferroptotic cancer cell death by GPX4. Cell 156, 317-331 (2014).

8. Lewerenz, J. et al. The cystine/glutamate antiporter system $\mathrm{x}(\mathrm{c})(-)$ in health and disease: from molecular mechanisms to novel therapeutic opportunities. Antioxid Redox Signal 18, 522-55 (2013).

9. Imai, H., Matsuoka, M., Kumagai, T., Sakamoto, T.\& Koumura, T. Lipid Peroxidation-Dependent Cell Death Regulated by GPx4 and Ferroptosis. Curr Top Microbiol Immunol 403, 143-170 (2017).

10. Louandre, C. et al. Iron-dependent cell death of hepatocellular carcinoma cells exposed to sorafenib. Int J Cancer 133, 1732-42 (2013). 
11. Louandre, C. et al. The retinoblastoma $(\mathrm{Rb})$ protein regulates ferroptosis induced by sorafenib in human hepatocellular carcinoma cells. Cancer Lett 356, 971-7 (2015).

12. Galmiche, A., Chauffert, B.\& Barbare, J.C. New biological perspectives for the improvement of the efficacy of sorafenib in hepatocellular carcinoma. Cancer Lett 346, 159-62 (2014).

13. Houessinon, A. et al. Metallothionein-1 as a biomarker of altered redox metabolism in hepatocellular carcinoma cells exposed to sorafenib. Mol Cancer 15, 38 (2016).

14. Feng, J. et al. ACSL4 is a predictive biomarker of sorafenib sensitivity in hepatocellular carcinoma. Acta Pharmacol Sin 42, 160-170 (2021).

15. Nie, J., Lin, B., Zhou, M., Wu, L.\& Zheng, T. Role of ferroptosis in hepatocellular carcinoma. $J$ Cancer Res Clin Oncol 144, 2329-2337 (2018).

16. Sun, X. et al. Activation of the p62-Keap1-NRF2 pathway protects against ferroptosis in hepatocellular carcinoma cells. Hepatology 63, 173-84 (2016).

17. Liang, C., Zhang, X., Yang, M.\& Dong, X. Recent Progress in Ferroptosis Inducers for Cancer Therapy. Adv Mater 31, e1904197 (2019).

18. Hassannia, B., Vandenabeele, P.\& Vanden Berghe, T. Targeting Ferroptosis to Iron Out Cancer. Cancer Cell 35, 830-849 (2019).

19. Bruix, J., Gores, G.J.\& Mazzaferro, V. Hepatocellular carcinoma: clinical frontiers and perspectives. Gut 63, 844-55 (2014).

20. Yang, C. et al. Gankyrin has an antioxidative role through the feedback regulation of Nrf2 in hepatocellular carcinoma. $J$ Exp Med 213, 859-75 (2016).

21. Chen, X., Yu, C., Kang, R.\& Tang, D. Iron Metabolism in Ferroptosis. Front Cell Dev Biol 8, 590226 (2020).

22. Liang, J.Y. et al. A Novel Ferroptosis-related Gene Signature for Overall Survival Prediction in Patients with Hepatocellular Carcinoma. Int J Biol Sci 16, 2430-2441 (2020).

23. Yang, Y. et al. RRM2 protects against ferroptosis and is a tumor biomarker for liver cancer. Cancer Cell Int 20, 587 (2020).

24. Sun, X. et al. Metallothionein-1G facilitates sorafenib resistance through inhibition of ferroptosis. Hepatology 64, 488-500 (2016).

25. Liu, T. et al. GPX2 overexpression indicates poor prognosis in patients with hepatocellular carcinoma. Tumour Biol 39, 1010428317700410 (2017).

26. Si, M.\& Lang, J. The roles of metallothioneins in carcinogenesis. J Hematol Oncol 11, 107 (2018).

27. Tao, Y.F. et al. Metallothionein III (MT3) is a putative tumor suppressor gene that is frequently inactivated in pediatric acute myeloid leukemia by promoter hypermethylation. $J$ Transl Med 12, 182 (2014).

28. Ding, C., Fan, X.\& Wu, G. Peroxiredoxin 1 - an antioxidant enzyme in cancer. J Cell Mol Med 21, 193-202 (2017).

29. Sun, Y.L. et al. Aberrant expression of peroxiredoxin 1 and its clinical implications in liver cancer. World J Gastroenterol 21, 10840-52 (2015).

30. Lv, X. et al. SRXN1 stimulates hepatocellular carcinoma tumorigenesis and metastasis through modulating ROS/p65/BTG2 signalling. J Cell Mol Med 24, 10714-10729 (2020).

31. Li, W., Lu, J., Ma, Z., Zhao, J.\& Liu, J. An Integrated Model Based on a Six-Gene Signature Predicts Overall Survival in Patients With Hepatocellular Carcinoma. Front Genet 10, 1323 (2019). 
32. Rao, Q.W. et al. Sulfiredoxin-1 is a promising novel prognostic biomarker for hepatocellular carcinoma. Cancer Med 9, 8318-8332 (2020).

33. Lovatt, M. et al. Peroxiredoxin-1 regulates lipid peroxidation in corneal endothelial cells. Redox Biol 30, 101417 (2020).

34. Zhu, J. et al. The Molecular Mechanisms of Regulating Oxidative Stress-Induced Ferroptosis and Therapeutic Strategy in Tumors. Oxid Med Cell Longev 2020, 8810785 (2020).

35. Yang, L. et al. Auranofin mitigates systemic iron overload and induces ferroptosis via distinct mechanisms. Signal Transduct Target Ther 5, 138 (2020).

36. Liu, Z. et al. Systematic Analysis of the Aberrances and Functional Implications of Ferroptosis in Cancer. iScience 23, 101302 (2020).

37. Zhou, N.\& Bao, J. FerrDb: a manually curated resource for regulators and markers of ferroptosis and ferroptosis-disease associations. Database (Oxford) 2020, (2020).

\section{Figure legends}

Figure 1. A flow chart for overall study design.

Figure 2. Upregulated and downregulated differentially expressed genes in hepatocellular carcinoma patients. (A) A heatmap showing the expressions of the 126 differentially expressed genes in the normal tissues and tumors of The Cancer Genome Atlas (TCGA) cohort. (B, C) Summary of gene mutation information in the (B) up-regulated genes and (C) down-regulated genes in the TCGA dataset. (D) The PPI network indicated the interactions among the differentially expressed genes.

Figure 3. The process of building the prognostic gene signature and signature-based risk score is a promising marker in TCGA and International Cancer Genome Consortium (ICGC) cohorts. (A) Distribution of LASSO coefficients of the 19 ferroptosis-related potential prognostic genes in training cohort. (B) The generated coefficient distribution plots for the logarithmic (lambda) sequence for the selection of the best parameter (lambda). (C) Forest plot showing the ferroptosis-related gene associated with the survival of patients with hepatocellular carcinoma. (D-I) The left represents the survival status of patients in the training (D) and validation (G) cohorts; the center represents the distribution of the risk score in the training $(\mathbf{E})$ and validation $(\mathbf{H})$ cohorts; the right represents the expression pattern of the prognostic signature genes in the classifiers of the high- and low-risk groups in the training (F) and validation (I) cohorts.

Figure 4. Kaplan-Meier analysis and time-dependent ROC analysis in training and validation cohorts. (A, D) The cut-off value in the TCGA (A) and ICGC (D) cohorts. (B, E) Kaplan-Meier overall survival (OS) curves for patients in the high-risk group and low-risk group in the TCGA (B) and ICGC (E) cohorts. (C, F) ROC curves showed the predictive efficiency of the risk signature for patients in the TCGA (C) and ICGC (F) cohorts on the survival rate.

Figure 5. Independent prognostic analysis of risk scores and clinical parameters. (A, B) The univariate 
and multivariate Cox regression analyses of the associations between the risk scores and clinical parameters and the OS of patients in the TCGA (A) and ICGC (B) cohorts.

Figure 6. Tumor-infiltrating immune cell (TIC) distribution map and the single-sample gene set enrichment analysis (ssGSEA) scores between different risk groups in the TCGA and ICGC cohorts. (A) The bar graph shows the relative content distribution of 19 TICs of hepatocellular carcinoma in the training cohort. (B) Boxplots of the correlations between the scores of 5 immune cells and risk groups in TCGA. (C) Boxplots of the correlations between the scores of 4 immune cells and risk groups in ICGC. (D) Boxplots of the correlations between 4 immune-related functions and risk groups in TCGA. (E) Boxplots of the correlations between the scores of 3 immune cells and risk groups in ICGC. Adjusted $\mathrm{p}$-values are shown as: $\mathrm{ns}$, not significant $(* \mathrm{p}<0.05 ; * \mathrm{*} p<0.01)$.

Figure 7. Gene Ontology (GO) and Kyoto Encyclopedia of Genes (KEGG) enrichment analysis in training and validation cohorts. (A, C) $\mathrm{GO}$ analysis based on prognostic ferroptosis-related genes in TCGA (A) and ICGC (C) cohorts. (B, D) KEGG pathway analysis based on prognostic ferroptosis-related genes in TCGA (B) and ICGC (D) cohorts.

Figure 8. The relations between FPI and tissues and different protein expression in four genes were verified in human tissue samples. (A) The different FPIs between tumor and normal samples among HCC. (B-E) Human Protein Atlas (HPA) immunohistochemistry using anti-GPX2 and anti-PRDX1 antibodies. Normal liver $(\mathbf{B}, \mathbf{C})$ vs. tumor tissues $(\mathbf{D}, \mathbf{E})$. 
Figures

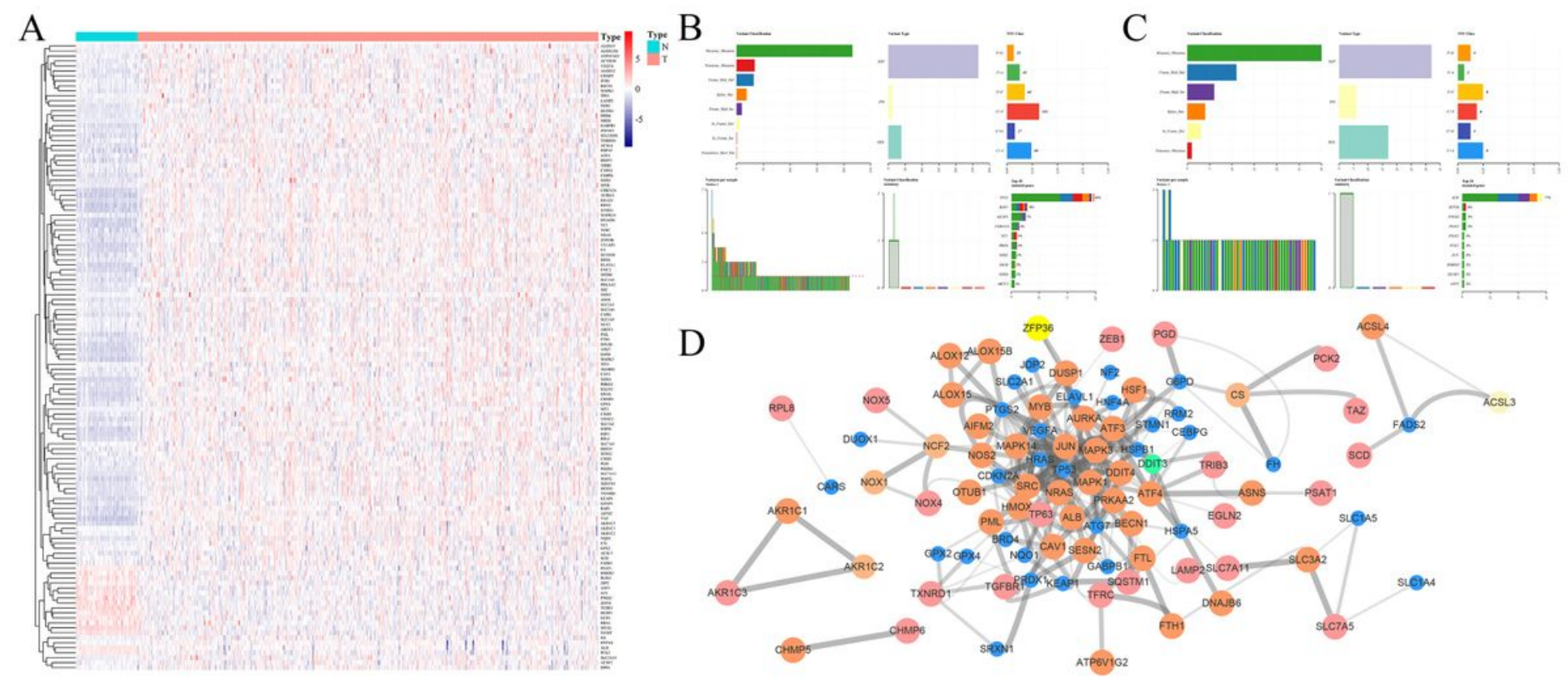

Figure 1

A flow chart for overall study design. 

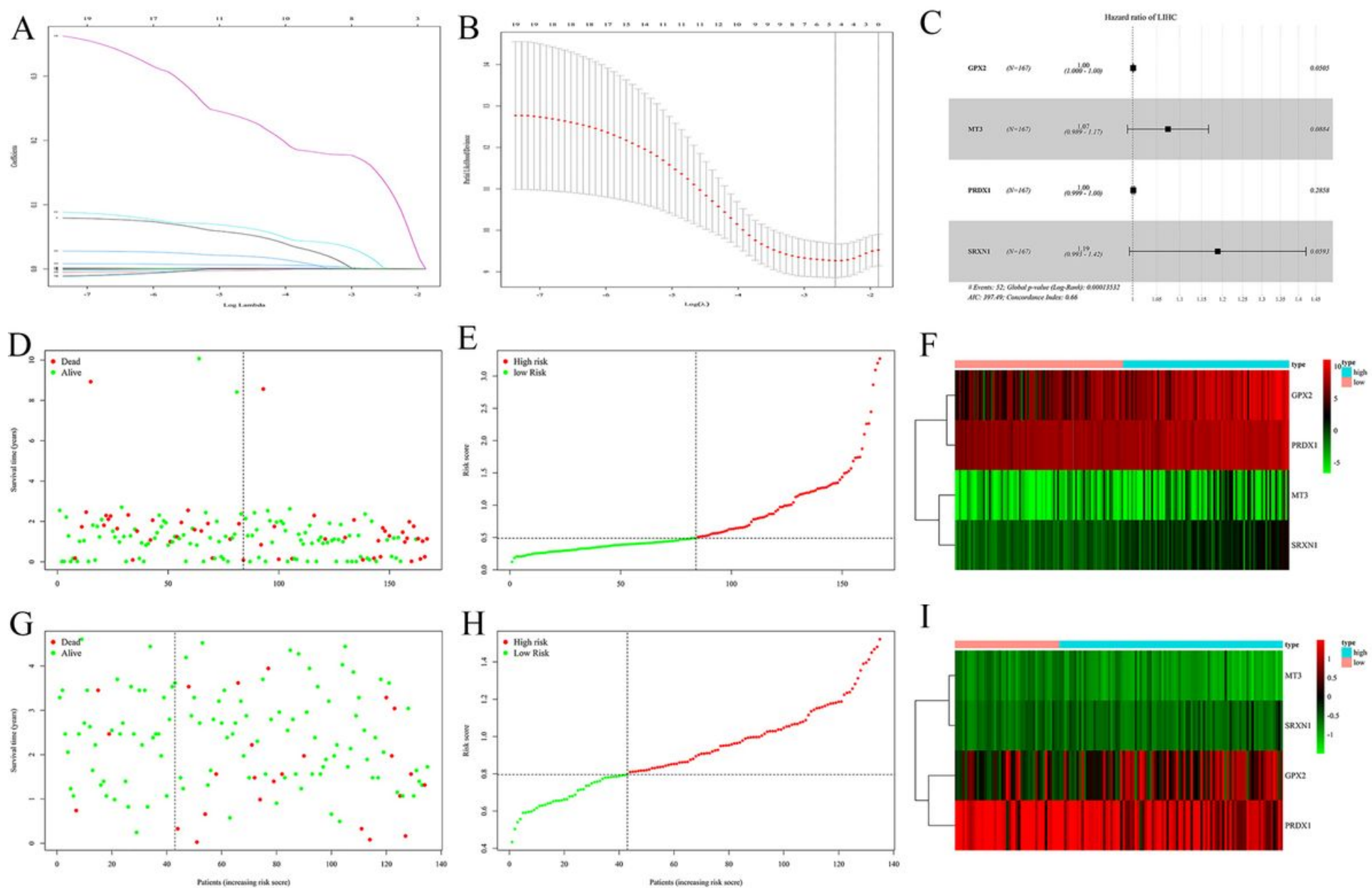

\section{Figure 2}

Upregulated and downregulated differentially expressed genes in hepatocellular carcinoma patients. (A) A heatmap showing the expressions of the 126 differentially expressed genes in the normal tissues and tumors of The Cancer Genome Atlas (TCGA) cohort. $(B, C)$ Summary of gene mutation information in the (B) up-regulated genes and (C) down-regulated genes in the TCGA dataset. (D) The PPI network indicated the interactions among the differentially expressed genes. 
A

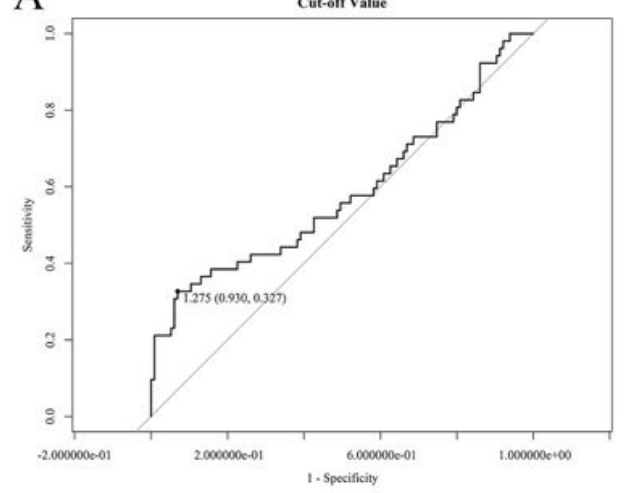

$\mathrm{D}$

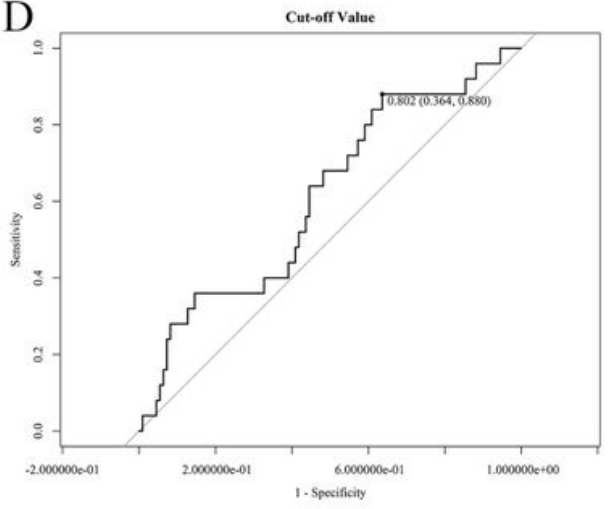

$\mathrm{B}$

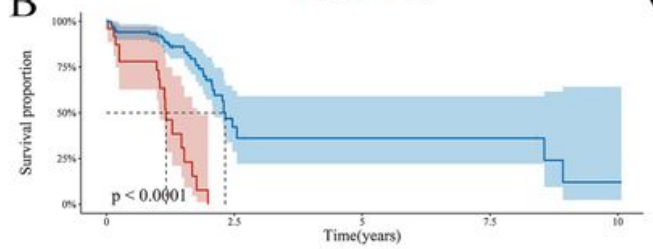

No.at risk

Init nat 25

Number of censosing
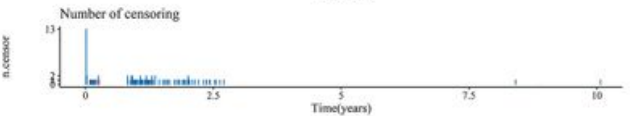

E

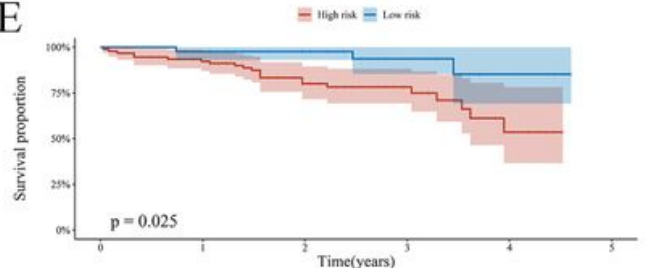

nemint

(
$\mathrm{C}$

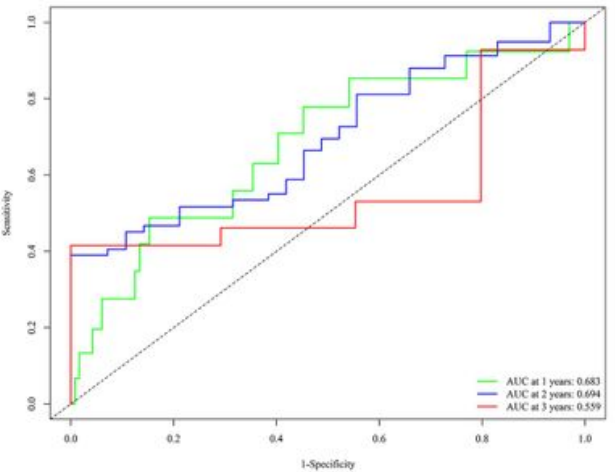

$\mathrm{F}$

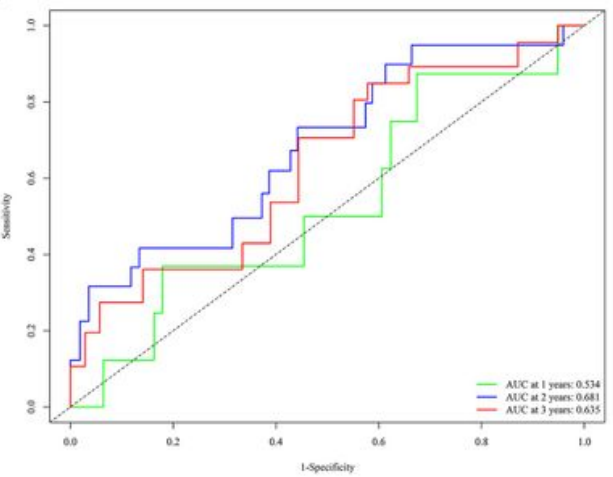

Figure 3

The process of building the prognostic gene signature and signature-based risk score is a promising marker in TCGA and International Cancer Genome Consortium (ICGC) cohorts. (A) Distribution of LASSO coefficients of the 19 ferroptosis-related potential prognostic genes in training cohort. (B) The generated coefficient distribution plots for the logarithmic (lambda) sequence for the selection of the best parameter (lambda). (C) Forest plot showing the ferroptosis-related gene associated with the survival of patients with hepatocellular carcinoma. (D-I) The left represents the survival status of patients in the training (D) and validation $(\mathrm{G})$ cohorts; the center represents the distribution of the risk score in the training $(\mathrm{E})$ and validation $(\mathrm{H})$ cohorts; the right represents the expression pattern of the prognostic signature genes in the classifiers of the high- and low-risk groups in the training $(F)$ and validation (I) cohorts. 
A

Age

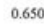

$1.005(0.983-1.027$

Gender

Stage

0.420

1. $143(0.826-1.581)$

riskScorc

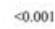

$2.728(1.849-4.027)$

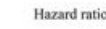

riskScore

$<.001$

$2.728(1.849-4.027$

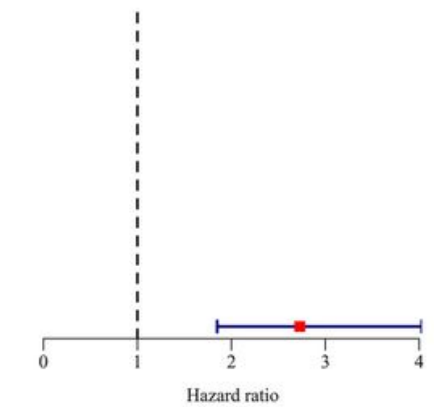

B
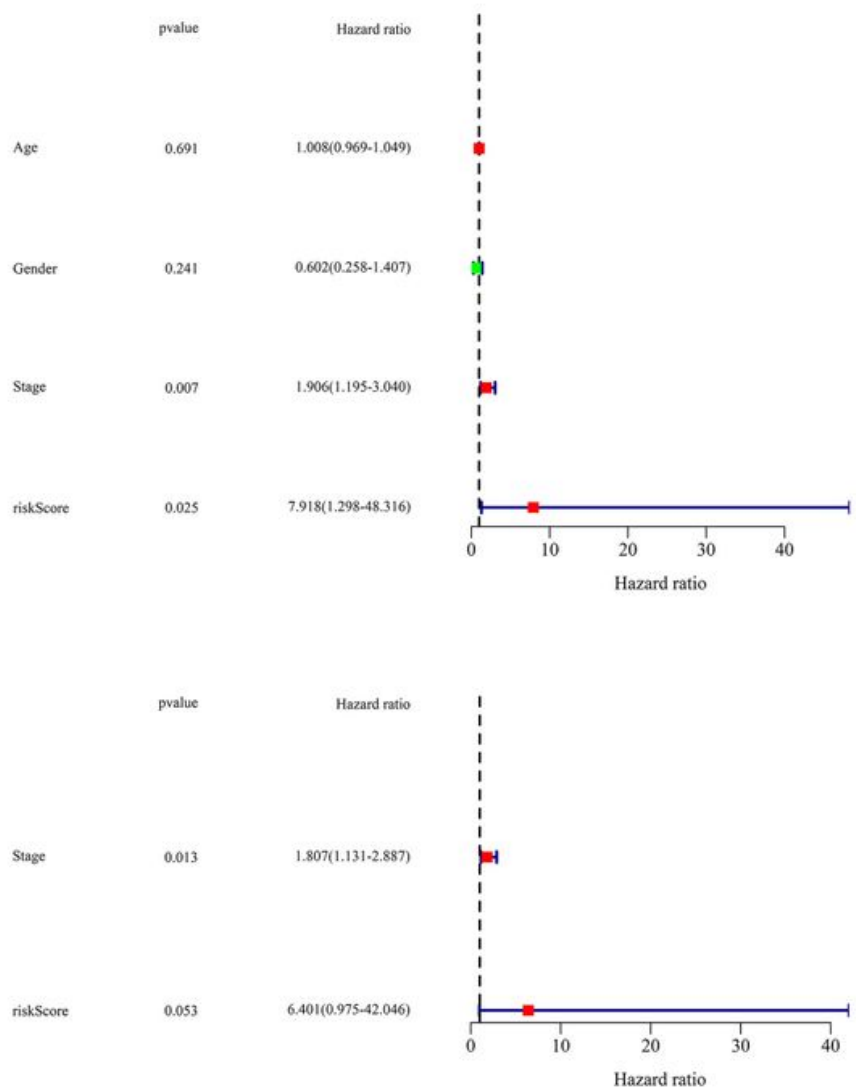

Figure 4

Kaplan-Meier analysis and time-dependent ROC analysis in training and validation cohorts. (A, D) The cut-off value in the TCGA (A) and ICGC (D) cohorts. (B, E) Kaplan-Meier overall survival (OS) curves for patients in the high-risk group and low-risk group in the TCGA (B) and ICGC (E) cohorts. (C, F) ROC curves showed the predictive efficiency of the risk signature for patients in the TCGA (C) and ICGC (F) cohorts on the survival rate.

A

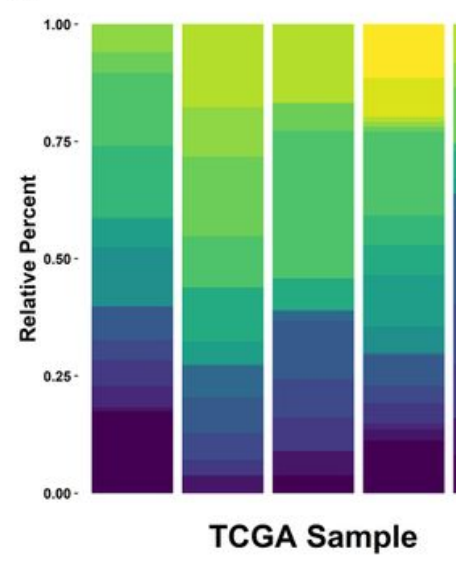

B

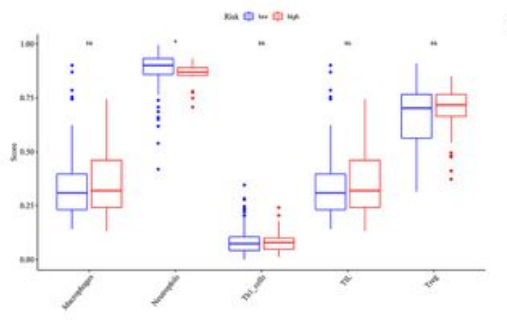

D

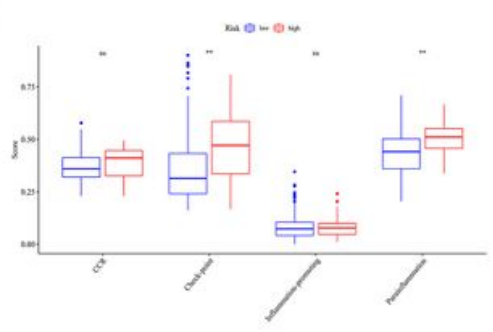

$\mathrm{C}$

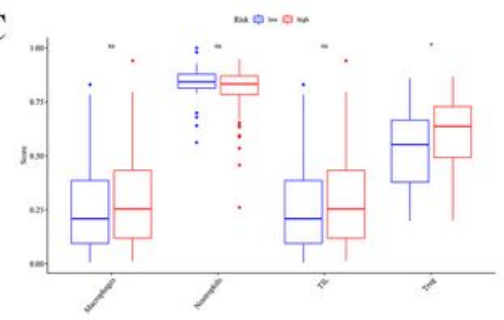

E

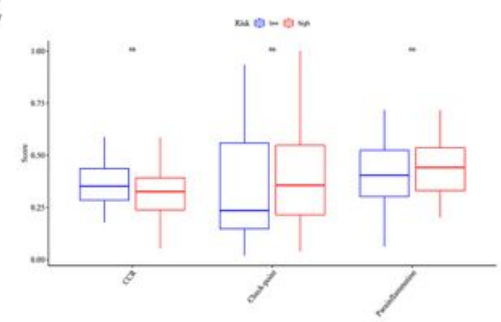

Figure 5 
Independent prognostic analysis of risk scores and clinical parameters. (A, B) The univariate and multivariate Cox regression analyses of the associations between the risk scores and clinical parameters and the OS of patients in the TCGA (A) and ICGC (B) cohorts.

A

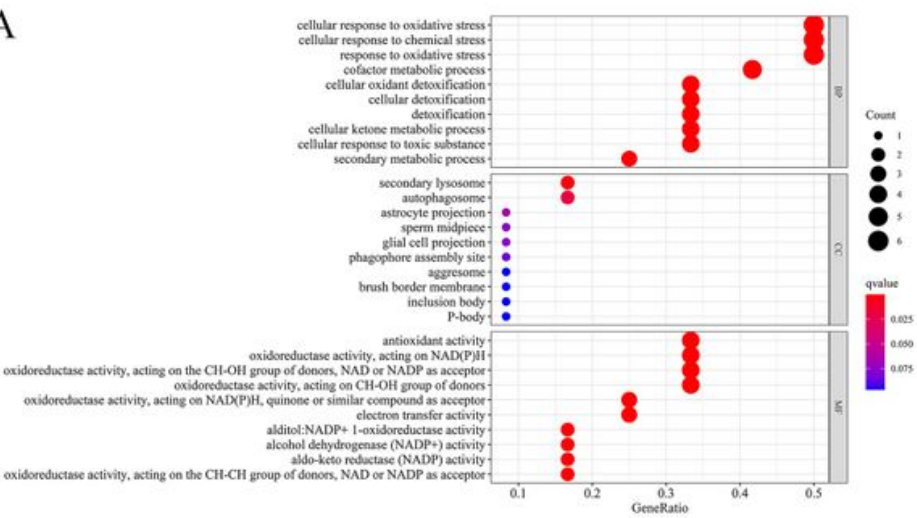

$\mathrm{C}$

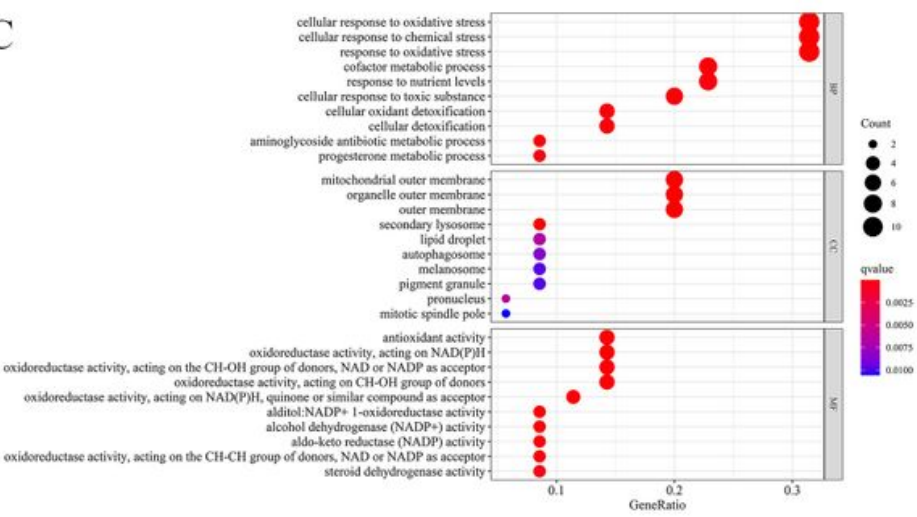

B

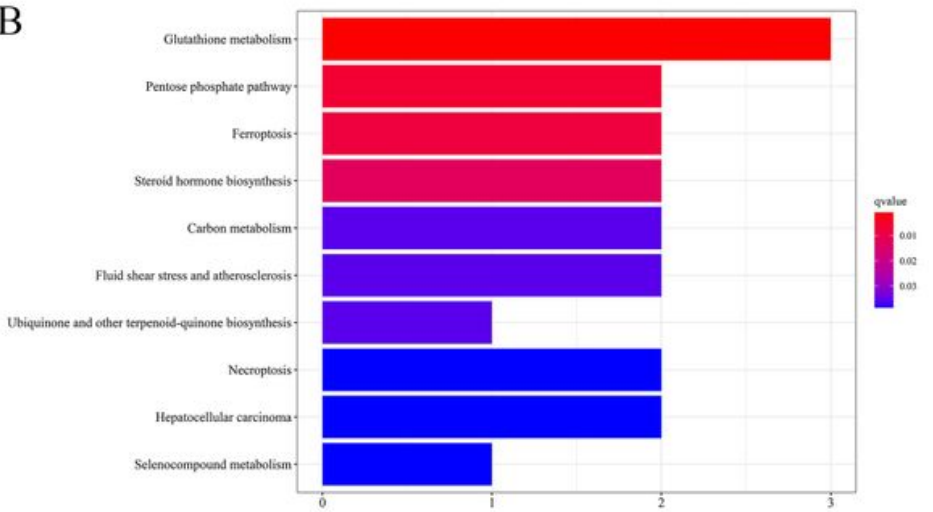

$\mathrm{D}$

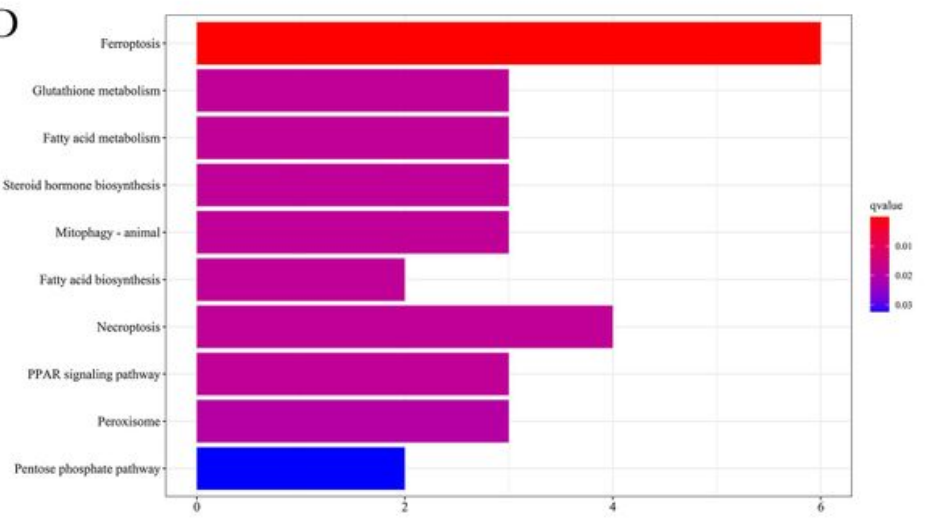

\section{Figure 6}

Tumor-infiltrating immune cell (TIC) distribution map and the single-sample gene set enrichment analysis (sSGSEA) scores between different risk groups in the TCGA and ICGC cohorts. (A) The bar graph shows the relative content distribution of 19 TICs of hepatocellular carcinoma in the training cohort. (B) Boxplots of the correlations between the scores of 5 immune cells and risk groups in TCGA. (C) Boxplots of the correlations between the scores of 4 immune cells and risk groups in ICGC. (D) Boxplots of the correlations between 4 immune-related functions and risk groups in TCGA. (E) Boxplots of the correlations between the scores of 3 immune cells and risk groups in ICGC. Adjusted p-values are shown as: ns, not significant $\left({ }^{\star} \mathrm{p}<0.05 ;{ }^{*} \mathrm{p}<0.01\right)$. 


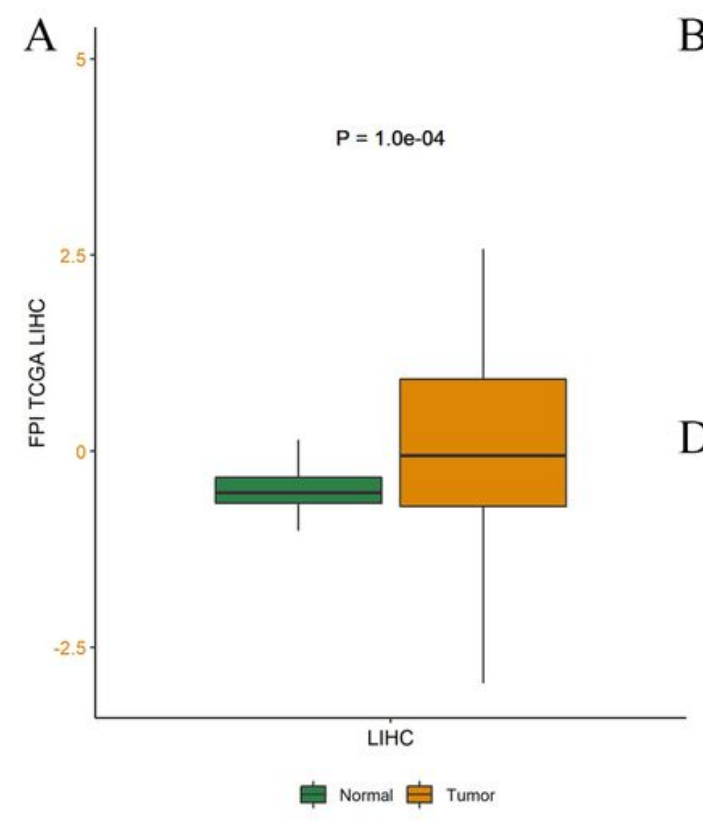

B

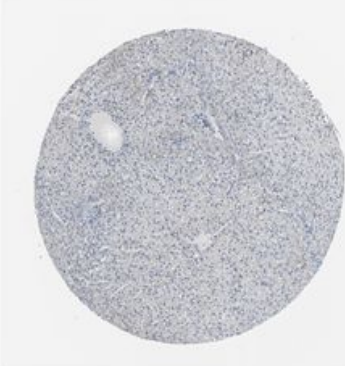

D

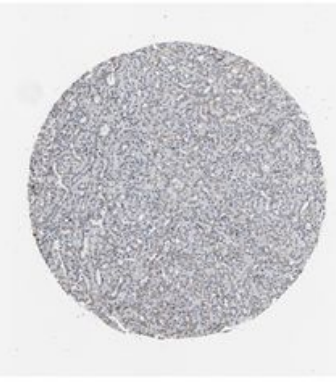

GPX2

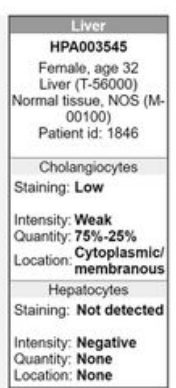

$\mathrm{C}$

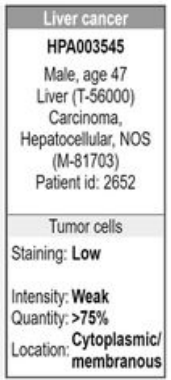

E

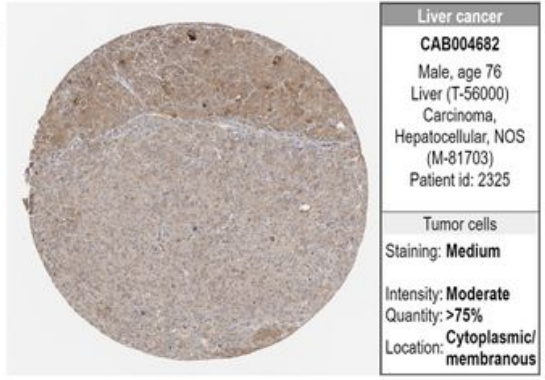

PRDX1

\section{Figure 7}

Gene Ontology (GO) and Kyoto Encyclopedia of Genes (KEGG) enrichment analysis in training and validation cohorts. (A, C) GO analysis based on prognostic ferroptosis-related genes in TCGA (A) and ICGC (C) cohorts. (B, D) KEGG pathway analysis based on prognostic ferroptosis-related genes in TCGA (B) and ICGC (D) cohorts.

\section{Figure 8 Placeholder}

Figure 8

The relations between FPI and tissues and different protein expression in four genes were verified in human tissue samples. (A) The different FPls between tumor and normal samples among HCC. (B-E) Human Protein Atlas (HPA) immunohistochemistry using anti-GPX2 and anti-PRDX1 antibodies. Normal liver $(B, C)$ vs. tumor tissues $(D, E)$. 